\title{
Vortex flow in a two-component unconventional superconductor
}

\author{
Qiang-Hua Wang \\ Department of Physics, University of Hong Kong, Pokfulam Road, Hong Kong, China \\ and Department of Physics and National Laboratory of Solid State Microstructure, Nanjing University, Nanjing 210093, China
}

Z. D. Wang

Department of Physics, University of Hong Kong, Pokfulam Road, Hong Kong, China

(Received 21 November 1997)

\begin{abstract}
Using a time-dependent two-component Ginzburg-Landau theory, we have studied the flow resistivity of vortices in the time-reversal-symmetry-breaking ( $T$-breaking) phases of unconventional superconductors. The free vortex flow resistivity is found to be generally nonlinear against the magnetic field. The relevance of these results in explaining a recent experiment by Wu et al. is addressed. [S0163-1829(98)01518-5]
\end{abstract}

Unconventional pairing is believed to most probably occur in heavy fermion superconductors such as $\mathrm{UPt}_{3} .{ }^{1} \mathrm{Re}-$ cently it was found that a Ru-based layered perovskite $\mathrm{Sr}_{2} \mathrm{RuO}_{4}$ shows the existence of superconductivity in the absence of copper. ${ }^{2}$ It seems probable that superconductivity in the Ru-based compound involves a different mechanism, via odd-parity pairing, from that operating in the copper oxides. $^{2-4}$ A more recent experiment on a $\mathrm{Ru}$-based double perovskite with copper doping, $\mathrm{Sr}_{2} \mathrm{YRu}_{1-x} \mathrm{Cu}_{x} \mathrm{O}_{6}$ with a transition temperature of about $30 \mathrm{~K}$, also points toward unusual superconducting properties. ${ }^{5}$ Apart from other anomalies, we mention only the magnetic-field-dependent resistivity. The $B(T)$ defined by the location of maximum $d R / d T$ in the $R-T$ curve at a field $B$ increases conventionally with decreasing temperature, but the $B(T)$ defined by vanishing resistance shows almost infinite slope at $T \sim 10 \mathrm{~K}$. Because of the intrinsic coexistence of ferromagnetism and superconductivity, the authors suspected that the Cooper pair may be of odd parity such as the $p$ wave. ${ }^{5}$

In this article, we present numerical results of one of the intrinsic macroscopic properties, the free vortex flow resistivity, in superconductors with odd-parity pairing. Although the field-dependent resistivity $\rho$ is complicated by the extrinsic nature of pinning in real samples, it approaches the intrinsic free vortex flow resistivity $\rho_{F}$ at high driving currents, and is related to $\rho_{F}$ in the thermally activated flux flow (TAFF) regime as $\rho=\rho_{F} \mu(T, B),{ }^{6}$ where $\mu(T, B)$ is the normalized mobility of the vortex in the pinning potential. ${ }^{7} \mathrm{We}$ find the following interesting behaviors of free vortex flow: (i) Axially symmetric vortices behave conventionally, irrespectively of the time-reversal-symmetry breaking ( $T$ breaking, hereafter); (ii) for the two kinds of nonaxial $T$-breaking vortices, only one type of them is stable under an applied current; (iii) the vortex flow resistivity is in general highly nonlinear in magnetic induction $B$. In particular, by assuming that the resistivity is measured in the TAFF regime, ${ }^{6}$ as is mostly the case in experiments, our results (see Figs. 1 and 2) are consistent with the anomalous zero-resistance $B(T)$ line of $\mathrm{Wu}$ et $a l^{5}$

The generic Ginzburg-Landau (GL) free energy functional is identical for singlet and triplet pairing, and is expressed as ${ }^{1}$

$$
\begin{aligned}
F= & \int d \Omega A(T)\left(\left|\eta_{1}\right|^{2}+\left|\eta_{2}\right|^{2}\right)+\beta_{1}\left(\left|\eta_{1}\right|^{2}+\left|\eta_{2}\right|^{2}\right)^{2} \\
& +\beta_{2}\left(\eta_{1}^{*} \eta_{2}-\eta_{1} \eta_{2}^{*}\right)^{2}+\beta_{3}\left|\eta_{1}\right|^{2}\left|\eta_{2}\right|^{2} \\
& +K_{1}\left(\left|D_{x} \eta_{1}\right|^{2}+\left|D_{y} \eta_{2}\right|^{2}\right)+K_{2}\left(\left|D_{x} \eta_{2}\right|^{2}+\left|D_{y} \eta_{1}\right|^{2}\right) \\
& +K_{3}\left(D_{x}^{*} \eta_{1}^{*} D_{y} \eta_{2}+\text { c.c. }\right)+K_{4}\left(D_{x}^{*} \eta_{2}^{*} D_{y} \eta_{1}+\text { c.c. }\right) \\
& +\frac{1}{8 \pi}\left(\mathbf{B}-\mathbf{H}_{e}\right)^{2},
\end{aligned}
$$

where $\left(\eta_{1}, \eta_{2}\right)(=\eta)$ are the two components of the order parameter in the real space, $\mathbf{D}=i \hbar \nabla-2 e \mathbf{A} / c, \mathbf{B}=\nabla \times \mathbf{A}$ is the local magnetic induction, and $\mathbf{H}_{e}$ is the applied magnetic field. As usual, $A(T) \sim \ln T / T_{c}$ defines the transition temperature. The other coefficients are material-dependent parameters. We note that if $K_{1}-K_{2}=K_{3}+K_{4}$ and $\beta_{3}=0$ the effective symmetry group is the $D_{6 h}$ system studied by Tokuyasu et al. ${ }^{8}$ On the other hand, if $K_{1}=K_{2}, K_{3}=K_{4}=0$, and $\beta_{3}=0$, Eq. (1) reduces to that of Machida et al. ${ }^{9}$

Assuming pure dissipative dynamics, we write the timedependent GL equations (TDGLE) as

$$
\partial_{t} \eta_{1,2}=-\Gamma \delta F / \delta \eta_{1,2}
$$

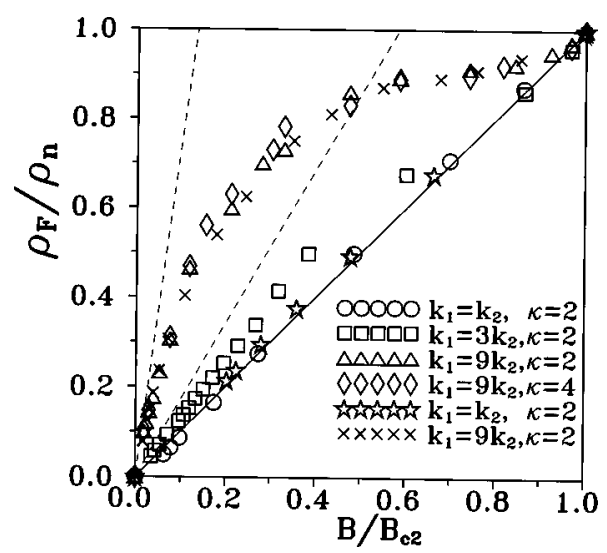

FIG. 1. Free vortex flow resistivity as a function of field. The solid line represents the linear law while the dashed lines are lowfield asymptotes. The open circles, squares, triangles, and diamonds are for square vortex lattices, while the crosses and open stars are for hexagonal vortex lattices. See the text for details. 


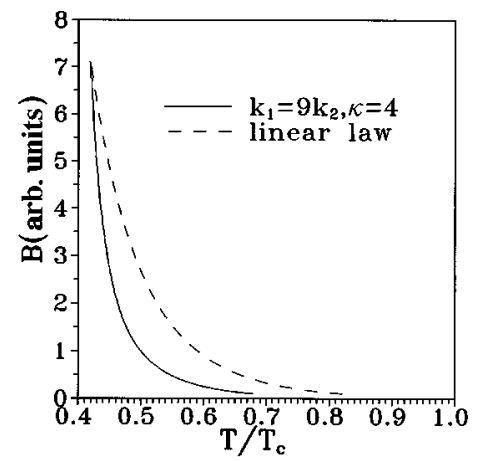

FIG. 2. Zero-resistance lines $B(T)$. The dashed line is obtained from the linear law and the solid line from the diamonds of Fig. 1. See the text for details.

$$
\partial_{t} \mathbf{A}=-c \rho_{n} \delta F / \delta \mathbf{A},
$$

where $\Gamma$ and $\rho_{n}=1 / \sigma_{n}$ are material constants and cannot be determined by the GL functional itself. $\Gamma$ determines the relaxation rate of the order parameters and $\rho_{n}\left(\sigma_{n}\right)$ is the normal state resistivity (conductivity). We are working in such a gauge that the electrostatic potential is absent. In Eq. (3) a fixed applied current $\mathbf{J}$ can be included by requiring $\nabla \times \mathbf{H}_{e}=(4 \pi / c) \mathbf{J}$. Since there is no a priori microscopic knowledge of $\Gamma$ for unconventional superconductors, we here make an ad hoc choice: $\Gamma=(2 e)\left(K_{1}+K_{2}\right) /\left\{\left[4\left(\beta_{1}\right.\right.\right.$ $\left.\left.\left.-\beta_{2}\right)+\beta_{3}\right] \sigma_{n}\right\}$, under which the TDGLE could lead to the usual linear vortex flow resistivity as a function of field $B$ (linear law hereafter) ${ }^{10,11}$ in our system when the timereversal symmetry is not broken.

We shall be interested in the case where $T$ breaking may be realized. This occurs for such $\left\{\beta_{i}\right\}$ that $\beta_{2}>0, \beta_{3}<4 \beta_{2}$, and $4\left(\beta_{1}-\beta_{2}\right)+\beta_{3}>0$. The $T$ breaking twofold degenerated bulk phase is $\left(\eta_{1}, \eta_{2}\right)=\eta_{0}(1, \pm i)$ with $\left|\eta_{0}\right|^{2}$ $=-A(T) /\left[4\left(\beta_{1}-\beta_{2}\right)+\beta_{3}\right]$. Following the convention, we define $\mathbf{q}=i \eta \times \eta^{*} /|\eta|^{2}$, which can be nonzero only if timereversal symmetry is broken, namely, $\eta$ cannot be transformed to $\eta^{*}$ by any gauge transform. Furthermore, define $\eta_{ \pm}=\left(\eta_{1} \pm i \eta_{2}\right) / \sqrt{2}\left|\eta_{0}\right|$. Then $\mathbf{q}=\left(\left|\eta_{-}\right|^{2}-\left|\eta_{+}\right|^{2}\right) \hat{z}$. The two pure $T$-breaking phases are the $\eta_{+}$phase (with $q$

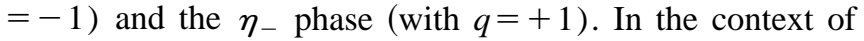
singlet (triplet) pairing, $q$ is proportional to the $z$-axis projection of the internal angular momentum (pseudospin) of the Cooper pair. ${ }^{1}$ In the presence of a magnetic field, both $T$-breaking phases could appear with one of them dominating the other. It was found that there exists two classes of $T$-breaking vortices in the $D_{6 h}$ system: $\mathrm{P}$ and AP vortices, where the $\mathrm{P}(\mathrm{AP})$ vortex is defined when $\mathbf{H}_{e}$ is parallel (antiparallel) to $\mathbf{q}$ of the dominant phase. ${ }^{8}$ When $K_{1} / K_{2} \neq 1$ the $\mathrm{P}$ vortex has the shape of a triangle while the AP vortex has the shape of a crescent. ${ }^{8,12}$ On the other hand, when $K_{1}$ $=K_{2}$ and $K_{3}=K_{4}=0$, both kinds of vortex are axially symmetric and energetically degenerated. ${ }^{8,9,12}$

Equations (2) and (3) are solved numerically by using the finite element method developed earlier. ${ }^{13,14}$ Without loss of generality, we constrain the parameters as follows: (i) $K_{1}$ $-K_{2}=K_{3}+K_{4}, \beta_{3}=0$, and (ii) $0<\widetilde{\beta_{2}} \equiv \beta_{2} /\left[4\left(\beta_{1}-\beta_{2}\right)\right.$ $\left.+\beta_{3}\right] \leqslant \beta_{c}$ with $\beta_{c} \sim 0.058$. For later convenience, let us define $k_{i}=K_{i} /\left(K_{1}+K_{2}\right), \quad \widetilde{k}=\left(k_{3}+k_{4}\right) / 2, \quad$ and $\quad \Delta \widetilde{k}=\left(k_{3}\right.$ $\left.-k_{4}\right) / 2$. The control parameters are $k_{1}$ and $\Delta \widetilde{k}$. In the weak coupling limit, $k_{1}=3 k_{2}=3 / 4$ and $\Delta \widetilde{k}=0,{ }^{1,15}$ But strong coupling effect would yield much more complicated situations. The upper critical field under condition (i) is $H_{c 2}^{I}=B_{0} /(1$ $-2 \Delta \widetilde{k})$ or $H_{c 2}^{I I}=B_{0} /\left[3-2 \sqrt{(1-\Delta \widetilde{k})^{2}+8 \widetilde{k}^{2}}\right]$, with the larger one as the relevant critical field. ${ }^{1}$ Here $B_{0}$ is a characteristic field scale $B_{0}=\Phi_{0} / 2 \pi \xi^{2}$, with $\xi$ $=\sqrt{-\left(K_{1}+K_{2}\right) / 2 A(T)}$ as a characteristic length scale similar to but maybe different to the coherence length. Another length scale similar to the London penetration depth is $\lambda=\sqrt{c /\left[8 \pi(2 e)\left(K_{1}+K_{2}\right)\left|\eta_{0}\right|^{2}\right]}$, so that an ad hoc GL parameter $\kappa$ can be defined as $\kappa=\lambda / \xi$. ${ }^{1}$ Condition (ii) ensures that the $T$-breaking vortex state is stabilized. ${ }^{8,9}$ We have performed extensive simulations for the driven $T$-violating vortices.

First, we consider $k_{1}=0.5=k_{2}\left(=1-k_{1}\right), \Delta \widetilde{k}=0$, and $\widetilde{\beta_{2}}=0.02$. In this case, $H_{c 2}=B_{c 2}=B_{0}$. We use $\kappa=2$ here, but essentially the same results are found for larger values of $\kappa$. The vortex structure turns out to be axially symmetric and has been described in detail by Machida et al. ${ }^{9}$ In short, (i) at extremely low fields $H_{e} \sim H_{c 1}$, when $\eta_{+}\left(\eta_{-}\right)$is the dominant phase, $\eta_{-}\left(\eta_{+}\right)$is localized and peaks at the core region of $\eta_{-}\left(\eta_{+}\right)$; (ii) at higher fields, the relative phases of $\eta_{1}$ and $\eta_{2}$ are locked (by the $\beta_{2}$ term in the free energy functional) as $\eta_{1}= \pm i \eta_{2}$ so that we have essentially either pure $\eta_{+}$or pure $\eta_{-}$phase. Since the P and AP vortices are energetically degenerated, the system behaves isomorphic to a conventional system and both $\mathrm{P}$ and AP vortices behave conventionally. This is exactly what we see in our simulation results. The open circles in Fig. 1 shows the field dependence of the free vortex flow resistivity $\rho_{F}$. It is seen to be essentially linear aside from minor numerical uncertainties. In particular, although the $\eta_{+}$phase may reside at the core region of the $\eta_{-}$phase (or vice versa) at fields near $H_{c 1}$, the system seems to still behave conventionally, a phenomenon we can trace back to the axial symmetry of the single vortex structure. The dashed line in Fig. 2 shows the "zero-resistance" $B(T)$ line in the presence of a model pinning potential when the linear law applies for $\rho_{F}$. In detail, we assume without loss of generality that $\rho_{n}(T) \propto T$, $B_{c 2}(T)=B_{c 2}(0) \sqrt{\left(1-t^{2}\right) /\left(1+t^{2}\right)}, \quad$ and $\quad \mu(T, B)=\exp$ $[-U(T, B) / T] \sim \exp \left[-u_{0}\left(1-t^{2}\right)^{3 / 2} / b^{s} t\right] \quad$ with $t=T / T_{c}$ and $b=B / B_{c 2}(0) .{ }^{7,16}$ Here $U(T, B)$ is the strength of the pinning potential. ${ }^{7}$ For general purpose we have assumed an inverse power law dependence of the pinning potential on the field with an exponent $s$. Then $\rho(T, B)=\rho_{F} \mu$. Let $u_{0}=2$ and $s$ $=0.1$. The maximum $T$ that matches a zero-resistance criterion $\rho(T, B) \leqslant \rho_{n}\left(T_{c}\right) / 100$ at a field $B$ is thus obtained. The zero-resistance $B(T)$ line for the linear law shows the desired steady increase of $B$ with decreasing $T$. The conventional behavior of axial $T$-breaking vortex flow is also reflected in the equilibrium magnetization curve (not shown here) extracted from the Virial theorem, ${ }^{17}$ with an extrapolated lower critical field $H_{c 1} \sim 0.173 B_{0}$, which is close to the conventional value $\left[\left(\ln \kappa / \kappa^{2}\right) H_{c 2}\right]$. Therefore neither magnetization nor vortex flow resistivity measurements could discriminate an axially symmetric $T$-breaking vortex state from conventional states. 


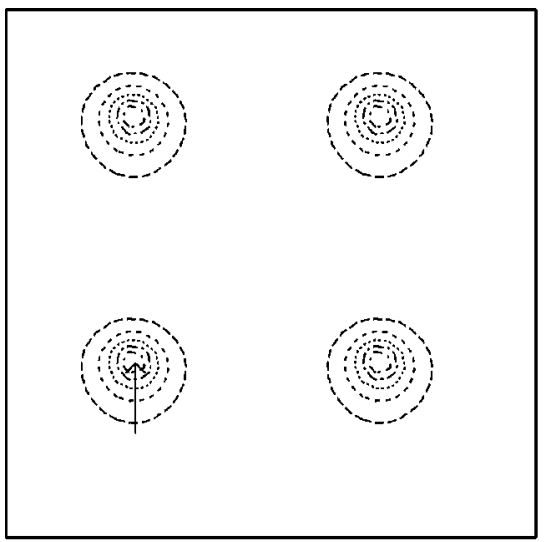

(a)

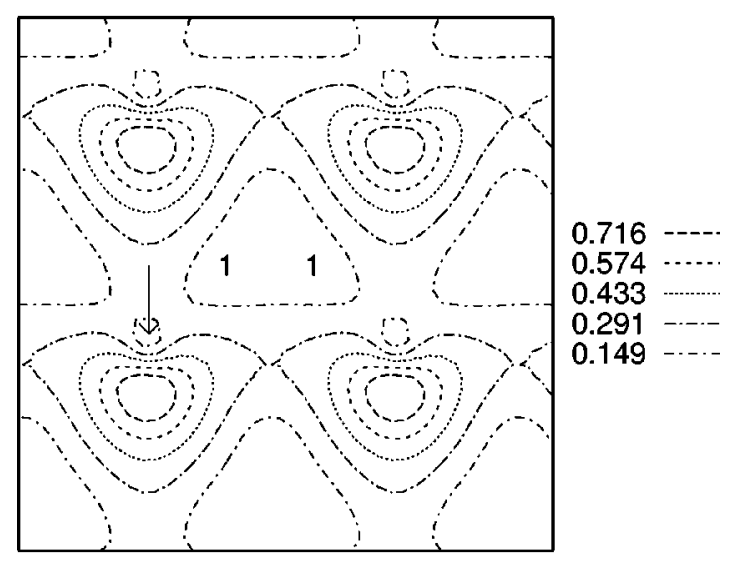

(b)

FIG. 3. Contour plots of (a) $\left|\eta_{+}\right| /\left|\eta_{0}\right|$ and (b) $\left|\eta_{-}\right| /\left|\eta_{0}\right|$ at $B$ $=\pi / 50 B_{0}$, obtained by replicating the $10 \xi \times 10 \xi$ unit cell twice in each direction. The arrows and the labels 1 denote the winding points. Note also the equivalent points.

Next, we consider $\kappa=2, k_{1}=3 k_{2}=0.75, \Delta \widetilde{k}=0, \widetilde{\beta}_{2}$ $=0.02$. In this case $H_{c 2}=H_{c 2}^{I I}=1.8165 B_{0}$ (while $H_{c 2}^{I}=B_{0}$ ). From the magnetization curve we extracted $H_{c 1} \sim 0.145 B_{0}$ $=0.0798 H_{c 2}$. The effective GL parameter is seen to be much larger than 2. Under a driving current, although both kinds of $T$-breaking vortices can move stably in the low-field regime $H \sim H_{c 1}$, only the AP vortex survives at higher fields. The $\mathrm{P}$-vortex state transforms to the AP-vortex state spontaneously. (If both $T$-breaking phases can coexist as we described below, we define a vortex to be the AP vortex in such a sense that the average of $\mathbf{q}$ is in the opposite direction of $\mathbf{H}_{e}$.) This is examined in two distinct ways. First, we set the initial state to be the normal state, and let the system evolve for a while by adding white Gaussian noises to the right hand sides of Eqs. (2) (a similar idea to the thermal annealing ${ }^{18}$ ). The noise is shut down in further evolution. And finally we always find the AP vortex. Second, we begin with a stabilized AP-vortex state and flip the direction of the magnetic field abruptly. In the low-field regime, this leads to the P-vortex state. But in the moderate field regime and under applied currents, we still obtain the AP-vortex state finally. (Of course, $\mathbf{q}$ is reversed automatically from its initial direction.) Since an extremely low field is not easily accessible to experiments, we only consider the AP-vortex motion. To be definite, let us assume that the applied magnetic field is positive. Then the dominant $T$-breaking phase in the AP-vortex state is the $\eta_{+}$
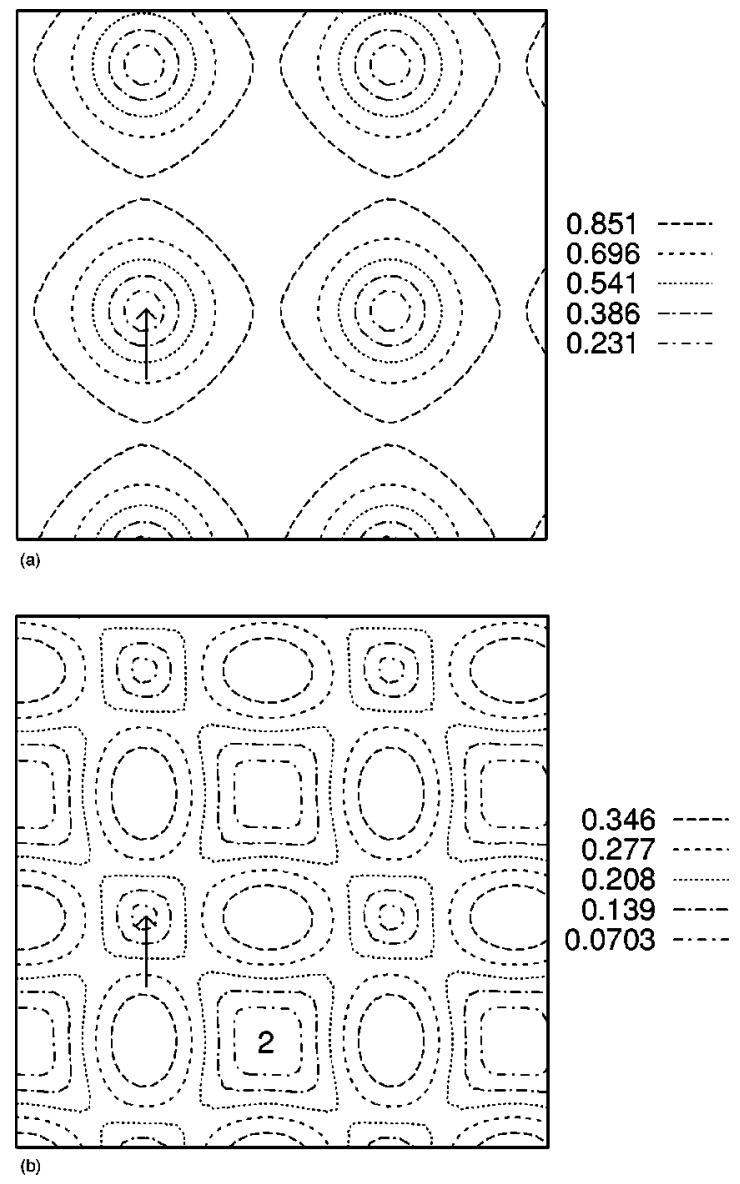

FIG. 4. The same plots as Figs. 3 except that $B=1.09 B_{0}$. The unit cell has dimensions $2.4 \xi \times 2.4 \xi$. The arrows and the label 2 denote the winding points.

phase. It is found that although the $\eta_{+}$phase is the dominant phase (see below), the $\eta_{-}$phase remains nonvanishing at all fields below $H_{c 2}$. This is in contrast to the situation of the axial vortices examined above. In Fig. 1 the open squares show the free AP-vortex flow resistivity as a function of the magnetic induction. As a consequence of the nontrivial coexistence of both $T$-breaking phases, the simplest linear law is clearly violated. The free flow resistivity is linear at low fields, but with a larger slope (see the dashed asymptote line) than what the simplest linear law would predict. At higher fields $B / B_{c 2}>0.3$ the resistivity deviates from the low-field asymptote gradually. The low-field asymptote line intersects with $\rho_{F} / \rho_{n}=1$ at approximately $B / B_{c 2}=H_{c 2}^{I} / H_{c 2} \sim 0.6$, suggesting the crossover behavior begins at the smaller upper critical field. To gain more insights into the anomalous crossover behavior, we present contour plots of $\eta_{ \pm}$at two representative fields in Figs. $3\left(B=\pi / 50 B_{0}\right)$ and Figs. 4 ( $B$ $\left.=1.09 B_{0}\right)$. For better visual quality, we have replicated the unit cell in our simulations twice in the $x$ (horizontal) and $y$ (vertical) directions. In Figs. 3 we see that the AP vortex is of a crescent shape as found earlier. ${ }^{8}$ On the other hand, in a unit cell the $\eta_{+}$phase has a single winding while $\eta_{-}$has three windings (cf. Ref. 8), with one of them being at the crescent front (see the arrow) and the other two being shown as labels 1 in Fig. 3(b). A closer inspection reveals that there is no shared winding point for $\eta_{ \pm}$phases in Figs. 3. This is because the separation of winding points lowers the kinetic 
energy. The field energy is also lifted but is negligible at low fields. Interestingly, by varying the direction of the applied current, we always find that the crescent front is directed along the moving direction of the AP vortex. At high fields, as in Figs. 4, the arrangement of the winding points changes. Since we have used a square vortex lattice geometry in the simulations, the profiles of $\eta_{ \pm}$are fourfold symmetric in Figs. 4. We have also used a rectangular unit cell with two flux quantum (in order to mimic hexagonal and other oblique vortex lattice structures) to find that there is always geometric frustration at low fields. The question of what should be the correct symmetry of the vortex lattice has been addressed by Tokuyasu et al. for the $\mathrm{P}$ vortices. ${ }^{8}$ Since the only symmetry of a single crescent vortex is a reflection symmetry, we believe that the assumption of a square lattice is reasonable but the lattice structure itself may deserve further investigations. The consequence of lattice geometry to the vortex polarity and the vortex flow resistivity will be examined below. Returning to our Figs. 4, we see that at this field, $\eta_{+}$and $\eta_{-}$share one winding point (see the arrows), evidently because at this level of field the energy gain in the kinetic part plays a less important role. On the other hand, the $\eta_{-}$phase has a twofold winding indicated by the label 2 in Fig. 4(b). Collecting the results at extremely low and high fields, we suspect that the structure of individual vortices plays an important role in the behavior of vortex flow.

The abnormal transport behavior of $T$-breaking vortices turns out to be rather generic at other points in the parameter space, provided that the aspect ratio $K_{1} / K_{2}\left(=k_{1} / k_{2}\right) \neq 1$ and $H_{c 2}^{I} \neq H_{c 2}^{I I}$. If $H_{c 2}^{I}<H_{c 2}^{I I}\left(H_{c 2}^{I}>H_{c 2}^{I I}\right)$ the AP (P) vortex is favored under an applied current. Since the behavior of the $\mathrm{P}$ vortex is similar to that of the AP vortex as found in our simulations, we concentrate on the behavior of the AP vortex. With increasing $H_{c 2}^{I I} / H_{c 2}^{I}$, the crossover regime in the flow resistivity is found to be broader. We present two such examples in Fig. 1 for $k_{1}=9 k_{2}=0.9, \Delta \widetilde{k}=0.1$, and $\widetilde{\beta}_{2}$ $=0.02$ at $\kappa=2$ (open triangles) and $\kappa=4$ (open diamonds). Here $H_{c 2}^{I}=1.25 B_{0}$ and $H_{c 2}^{I I}=9.205 B_{0}$. Apart from the generic feature just mentioned, we see that the low-field asymptote seems to be independent of $\kappa$. The shape of a knee (with a plateau at high fields) in the crossover is now obvious. Moreover, the low-field asymptote intersects with $\rho_{F} / \rho_{n}=1$ at $B / B_{c 2} \sim 0.14$, again corresponding to the smaller upper critical field $H_{c 2}^{I}$. It seems likely that the vortex flow resistivity deviates significantly from the low-field asymptote beyond the smaller upper critical field at which winding-point separation is no longer energetically favorable.

At this stage, it seems pertinent to mention to what extent the above results for square vortex lattices are generic for other geometries. For this purpose, in Fig. 1 we have also presented vortex flow resistivity for hexagonal lattices (crosses and stars). We note that the polarity of the dynamically stable vortices is the same as in the hexagonal lattice. Evidently, from Fig. 1 the free flow resistivity is not at all as sensitive to the geometry as one would have expected. However, this is reasonable in that the interaction between the vortices at low fields is weak while the vortices (for the dominant phase) undergo core transitions to be locally axial at high fields for any geometry.

Concluding Fig. 1, we observe that the nonlinear behavior of the resistivity is a rather general feature of superconductors with multiple-component unconventional pairing. We suspect that such a highly nonlinear behavior might have been observed in the recent field-dependent resistivity measurements of $\mathrm{Wu}$ et al. in the $\mathrm{Sr}_{2} \mathrm{YRu}_{1-x} \mathrm{CuO}_{6}$ system (carrying a possible odd-parity pairing state with $\left.T_{c} \sim 30 \mathrm{~K}\right){ }^{5}$ To bear out this conjecture, we first fit the diamonds in Fig. 1 by a polynomial function, $\rho_{F}=\rho_{n}(T) f\left[B / H_{c 2}(T)\right]$. Using the same choices for $\rho_{n}(T), H_{c 2}(T), \mu(T, B)$ and the zeroresistance criterion as those used for the dashed line in Fig. 2, we obtain the zero-resistance $B(T)$ line, the solid line in Fig. 2, in connection with nonlinear vortex flow resistivity. Evidently the solid line agrees better with Fig. 4 of Ref. 5 qualitatively because of the crossover behavior of the highly nonlinear vortex resistivity.

We acknowledge the support from a RGC grant of Hong Kong and the National Center for Research and Development on Superconductivity of China.
${ }^{1}$ M. Sigrist and K. Ueda, Rev. Mod. Phys. 63, 239 (1991).

${ }^{2}$ Y. Maeno et al., Nature (London) 372, 532 (1994).

${ }^{3}$ T. Oguchi, Phys. Rev. B 51, 1385 (1995); D. J. Singh, ibid. 52, 1358 (1995).

${ }^{4}$ T. M. Rice and M. Sigrist, J. Phys.: Condens. Matter 7, L643 (1995); for another viewpoint, see, e.g., D. F. Agterberg, T. M. Rice, and M. Sigrist, Phys. Rev. Lett. 78, 3374 (1997).

${ }^{5}$ M. K. Wu et al., Z. Phys. B 102, 37 (1997).

${ }^{6}$ E. H. Brandt, Rep. Prog. Phys. 58, 1465 (1995); Z. D. Wang et al., Supercond. Sci. Technol. 9, 333 (1996).

${ }^{7}$ M. Tinkham, Phys. Rev. Lett. 61, 1658 (1988).

${ }^{8}$ T. A. Tokuyasu et al., Phys. Rev. B 41, 8891 (1990).

${ }^{9}$ K. Machida et al., J. Phys. Soc. Jpn. 62, 680 (1993).

${ }^{10}$ J. Bardeen and M. Stephen, Phys. Rev. A 140, 1197 (1965); M. Stephen and J. Bardeen, Phys. Rev. Lett. 14, 112 (1965).
${ }^{11}$ See, e.g., A. I. Larkin and Yu. N. Ovchinnikov, in Nonequilibrium Superconductivity, edited by D. N. Langenberg and A. I. Larkin (North-Holland, Amsterdam, 1986).

${ }^{12}$ Z. D. Wang and Q. H. Wang, Phys. Rev. B 57, R724 (1998).

${ }^{13}$ Q. Du, M. D. Gunzburger, and J. S. Peterson, Phys. Rev. B 51, 16 194 (1995).

${ }^{14}$ Q. Wang and Z. D. Wang, Phys. Rev. B 54, R15 645 (1996); Z. D. Wang and Q. H. Wang, ibid. 55, 11756 (1997).

${ }^{15}$ J.-X. Zhu et al., Phys. Rev. B 56, 14093 (1997).

${ }^{16}$ M. Tinkham, Introduction to Superconductivity (McGraw-Hill, New York, 1996).

${ }^{17}$ M. M. Doria et al., Phys. Rev. B 39, 9573 (1989); Z. D. Wang and C.-R. Hu, ibid. 44, 11918 (1991).

${ }^{18}$ M. M. Doria et al., Phys. Rev. B 41, 6335 (1990). 